\title{
Inconsistencias de la Terminologia Anatomica: La Vértebra Cervical 1 Nombrada con el Epónimo Atlas
}

\author{
Inconsistencies of the Terminologia Anatomica: \\ First Cervical Vertebra Named with the Eponim Atlas
}

Jorge Eduardo Duque Parra ${ }^{1,2}$; John Barco Ríos ${ }^{1}$ \& Juan Fernando Vélez García ${ }^{3}$

\begin{abstract}
DUQUE, J. E. P.; BARCO, J. R. \& VÉLEZ, J. F. G. Inconsistencias de la Terminologia Anatomica: La vértebra cervical 1 nombrada con el epónimo Atlas. Int. J. Morphol., 37(2):412-415, 2019.

RESUMEN: La primera vértebra cervical establece una articulación sinovial con los cóndilos del occipital, sin que haya de por medio disco fibrocartilaginoso. Esta articulación es importante para la anatomía funcional del cuello porque, además de soportar el peso de la cabeza en los humanos, permite movimientos de flexión, extensión e inclinación lateral. La primera vértebra es conocida en la Terminologia Anatomica y en la Nomina Anatomica Veterinaria con el nombre de Atlas [C1]. El nombre de Atlas, asignado a la primera vértebra cervical, fue tomado de uno de los titanes de la mitología griega condenado por el dios Zeus a soportar el cielo con sus manos, cabeza y hombros, y que en términos figurativos es la que sostiene el mundo craneal. Sorprende que en el libro oficial de la Terminologia Anatomica y en el de la Nomina Anatomica Veterinaria se mantenga este epónimo por una simple tradición, constituyéndose en una vergonzosa contradicción que debe ser corregida y eliminada.
\end{abstract}

PALABRAS CLAVE: Atlas; Vértebra cervical; Terminologia Anatomica; Epónimos.

\section{INTRODUCCIÓN}

En el libro oficial de Terminologia Anatomica se le denomina Atlas [C1] a la primera vértebra cervical (Fig. 1), identificada además con el código alfanumérico A02.2.02.101 (Federative Committe on Anatomical Terminology, 1998), mientras que en Nomina Anatomica Veterinaria solo se le denomina Atlas, sin el sinónimo C1 (International Committee on Veterinary Gross Anatomical Nomenclature, 2017). Esta vértebra es fundamental para la anatomía funcional de la columna, y su diseño presenta notables diferencias con el de otras vértebras, como las de la columna lumbar (Manfreda et al., 2006). En la columna cervical, la vértebra C1 (Fig. 1) establece una articulación sinovial con los cóndilos del occipital, sin presencia de disco fibrocartilaginoso. Mediante esta articulación se permite el movimiento de rotación cérvicocapital (Duque Parra et al., 2010) y del gesto del "si", así como la inclinación lateral de la cabeza (Vargas Sanabria, 2012), pero además permite soportar y transmitir la carga de la cabeza a las vértebras cervicales subyacentes (Bogduk, 2016). El peso de la cabeza es es- tabilizado por las dos masas laterales, las cuales están conectadas por los arcos anterior y posterior (Kayalvizhi et al., 2017), que en cuadrúpedos se les denomina arcos ventral y dorsal (Dyce et al., 2012; ICVGAN, 2017), y junto con su articulación al axis permite movimientos amplios de la cabeza (Dyce et al.). En mamíferos domésticos esta vértebra presenta gran variedad de formas $y$, a diferencia del humano, está conformada por unos procesos transversos más desarrollados, por lo que también se les ha denominado como alas del atlas [Ala atlantis] (ICVGAN).

La vértebra cervical uno, en humanos, puede presentar algunas variantes anatómicas, entre las que se destacan las siguientes: hipoplasias (Yamahata et al., 2017), ausencia de osificación del arco posterior debida a falla en la fusión de la línea mediana de los dos semiarcos vertebrales posteriores (Sánchez-García \& Caro, 2014), agenesia parcial del arco posterior que afecta su estabilidad (Roa, 2015) y, en consecuencia, la estabilidad del cráneo.

\footnotetext{
${ }^{1}$ Universidad de Caldas, Programa de Medicina, Departamento de Ciencias Básicas, Manizales, Colombia.

${ }^{2}$ Universidad de Manizales, Programa de Medicina, Departamento de Ciencias Básicas, Manizales, Colombia.

${ }^{3}$ Universidad del Tolima, Facultad de Medicina Veterinária y Zootecnia, Departamento de Sanidad Animal, Ibagué, Colombia.
} 


\section{DISCUSIÓN}

En el siglo VIII a.n.e, el poeta griego Hesíodo, contemporáneo de Homero, en su obra Teogonía, realiza una narración completa del origen de los dioses y titanes griegos. Hesíodo cuenta cómo el dios griego Zeus tuvo que luchar contra sus hijos los titanes y sus aliados. Después de la batalla por la dominación del mundo, Zeus, victorioso, condenó a uno de los titanes, el gigante Atlas $\omega t \lambda \alpha \zeta$ (Fig. 2), a sostener y soportar el gran cielo con la cabeza y los brazos (Hesíodo, 2010; Karemberg, 2012). En el siglo I a.n.e, el enciclopedista romano Plinio el viejo, empleó el término atlantion -que corresponde al diminutivo de Atlas- para referirse al segmento cervical craneal del esqueleto de la hiena -Hyaena hyaena-. Tres siglos después, Julius Pollux -Pollucis-, reconocido experto en terminología antigua, utilizó el término Atlas para designar a la séptima vértebra cervical de los humanos (Pollucis, 1824), pues, según él, este hueso permitía llevar cargas sobre los hombros. El asentamiento de un gran peso sobre la región posterior del cuello se soporta principalmente sobre el proceso espinoso de la séptima vértebra cervical, no sobre la primera vértebra que articula con el occipital. En mamíferos cuadrúpedos la posición y forma del atlas cambian la biomecánica; por lo tanto, la musculatura es más desarrollada y se fija a los grandes procesos transversos que tienen (Liebich \& König, 2005), pero aun así cuando el animal mantiene la cabeza en lo alto, el peso se transmite a la última vértebra cervical (C7) (Fig. 3).

Cerca de 1600 años después de Plinio el viejo, el término Atlas fue definitivamente establecido como referencia para la vértebra cervical superior $\mathrm{C} 1$, que en términos figurativos es la que carga el "mundo" craneal (Fig. 2). Sin embargo, en casos excepcionales, como sucede en la sinostosis atlanto-occipital, la carga es parcial (Duque Parra et al., 2018) ya que el peso del cráneo será soportado por la segunda vértebra cervical. En general, la columna vertebral cervical sostiene y equilibra el peso de la cabeza mediante la acción permanente de músculos, tendones y ligamentos. Galeno de Pérgamo (130-210) llamó a esta vértebra protosponio -del griego protos primero y spondylos espina-, más fue sólo a mediados del siglo XVI que se pasó a denominar Atlas, aunque en el texto de la época de Andreas Vesalio (1514-1564) aparece como primera vértebra cervical (Vesalius, 1555) y en la literatura española del mismo siglo se menciona a $\mathrm{C} 1$ como el primer hueso del pescuezo (de Valverde de Amusco, 1556).

Así pues, el nombre de Atlas para la vértebra C1 fue tomado del titán de la mitología griega (Riascos Catañeda \& Castro Navarro, 2004), lo que resulta ser un



Fig. 1. Vista superior de la primera vértebra cervical humana.

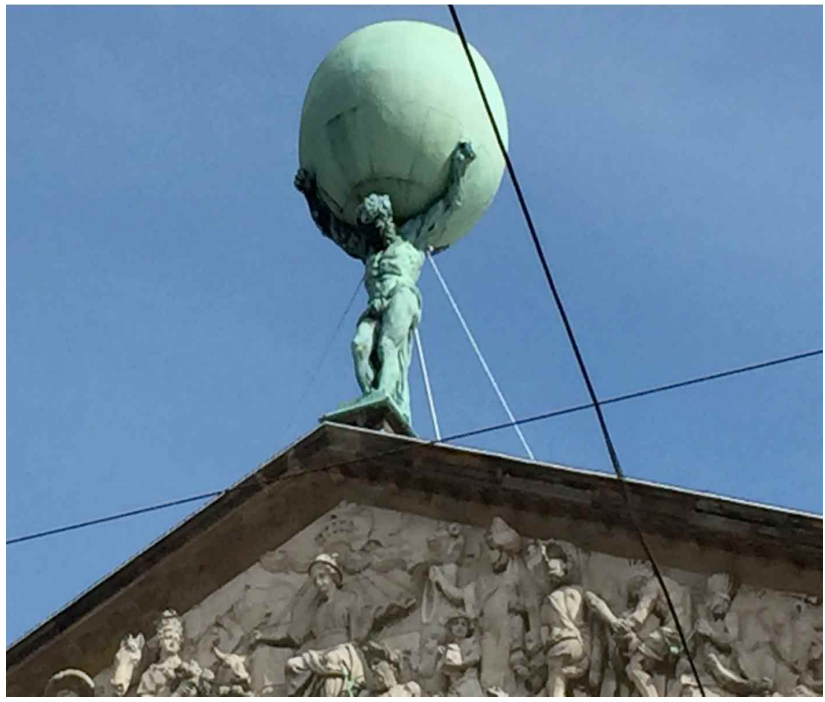

Fig. 2. Estatua del coloso Atlas sosteniendo el mundo sobre sus hombros y en la parte posterior de su cuello, recibiéndolo parte del peso sobre el proceso espinoso de la vértebra cervical 7.



Fig. 3. Vértebras cervicales de ballena, nótese en la parte craneal C1. 
epónimo, razón por la cual no debió incluirse en la Terminologia Anatomica ni en Nomina Anatomica Veterinaria, como un legado del siglo XVI y no como un término adecuado no epónimo ya usado desde Plinio el Viejo, pasando por Galeno y hasta Vesalio -término que duró unos 1600 años-, para pasar a ser un epónimo que se ha mantenido por 400 años. Quizá se trata de una visión romántica de los anatomistas que decidieron este nombre en las reuniones desde finales del siglo XIX. Sin embargo, ellos, reacios a nombrar las estructuras empleando una lógica morfológica o funcional, adoptaron el término Atlas de la mitología, nombre que ha permanecido impasible e inmutable desde ese tiempo, a pesar de estar violando el precepto establecido de no usar epónimos en la descripción anatómica. Incluso, el término sigue apareciendo en la última edición (sexta) de Nomina Anatomica Veterinaria (ICVGAN), siendo una versión más actual que la Terminologia Anatomica. Los epónimos, en general, no dan significado de la naturaleza de una estructura (Duque \& Ríos, 2013) ni de su función (Duque-Parra et al., 2018), tampoco transmiten los hallazgos característicos asociados con la enfermedad o estructura con la que se asocian (Fargen \& Hoh, 2014). En otras palabras, los epónimos no brindan ninguna información relevante que permita orientarnos y saber de qué estructura se trata, llevando al desconocimiento de lo que dicen otros (Duque Parra et al., 2012).

Terminologia Anatomica, como cualquier lenguaje vivo, ha estado sujeta a modificaciones, adiciones y eliminaciones (Batigália et al., 2015) durante más de 20 siglos, donde muchos de estos cambios se han dado sin una razón científica evidente (van Dijk et al., 2011), solo por consenso general. Este caso del Atlas, es otro caso entre muchos, donde se hace evidente algunas contradicciones en las que incurren las personas que hacen ciencia, por el solo hecho de ceñirse a una tradición dogmática. La ciencia per se es dinámica y renovadora, pero la naturaleza humana gusta de la tradición y el dogmatismo.

\section{CONCLUSIÓN}

En el libro oficial de Terminologia Anatomica y en Nomina Anatomica Veterinaria aparece el término Atlas para denominar a la primera vértebra cervical, un hecho que va en contraposición con la directriz terminológica de no utilizar epónimos para nombrar las estructuras corporales, sin importar de dónde procedan. Esto evidencia que aun existe cierto arraigo por la terminología epónima como incongruencia de la ciencia anatómica.
DUQUE, J. E. P.; BARCO, J. R. \& VÉLEZ, J. F. G. Inconsistencies of the Terminologia Anatomica: First cervical vertebra named with the eponim Atlas. Int. J. Morphol., 37(2):412415, 2019

SUMMARY: The first cervical vertebra establishes a synovial joint with the occipital condyles, without having a fibrocartilaginous disc. This joint articulation is important for the functional anatomy of the neck, because in addition to supporting the weight of the head in humans, it allows movements of flexion, extension and lateral inclination. The first cervical vertebra is known in the Terminologia Anatomica and the Nomina Anatomica Veterinaria with the name of Atlas [C1]. The name Atlas, assigned to the first cervical vertebra, was taken from one of the titans of Greek mythology condemned by the god Zeus to support the world with the hands, head, and shoulders, so in figurative terms said vertebra is the one that sustains the cranial world. It is surprising that in the official book of the Terminolgia Anatomica and in the Nomina Anatomica Veterinaria this eponymous is maintained by a simple tradition, constituting a shameful contradiction that must be corrected and eliminated.

KEY WORDS: Atlas; Cervical vertebra; Terminology; Eponymous.

\section{REFERENCIAS BIBLIOGRÁFICAS}

Batigália, F.; Boer, N. P.; Marcatto, G. \& Boer, A. L. R. Applicability and critical analysis of the use of eponyms in Health Sciences. J. Morphol. Sci., 32(4):264-6, 2015.

Bogduk, N. Functional anatomy of the spine. Handb. Clin. Neurol., 136:67588, 2016.

de Valverde de Amusco, J. Historia de la Composición del Cuerpo Humano. Roma, Antonio Salamanca y Antonio Lafrerii, 1556.

Duque Parra, J. E.; Barco Ríos, J. \& Moscoso Ariza, O. H. Degrees of Cervico-capital rotation of the Caldas people, Colombia. Int. J. Morphol., 28(4):1125-9, 2010.

Duque Parra, J. E.; Barco Ríos, J. F. \& García Aguirre, J. F. Atlanto-occipital synostosis in a Colombian population sample. J. Morphol. Sci., 35(1):28-30, 2018.

Duque Parra, J. E.; Barco Ríos, J. \& Duque Quintero, N. Refining the Anatomical Terminology: opening and foramen, two similarities and redundancy. Int. J. Morphol., 30(4):1497-9, 2012.

Duque-Parra, J. E.; Barco-Ríos, J. \& Dávila-Alzate, N. Los epónimos en las ciencias médicas: errores históricos que originan injusticias. Rev. Fac. Med., 66(1):87-90, 2018.

Duque, J. E. \& Ríos, J. Umbilical-spinous line: a morphological term that should be included in the anatomical terminology. Colomb. Med. (Cali), 44(3):202-4, 2013.

Dyce, K. M.; Sack, W. O. \& Wensing, C. J. G. Anatomía Veterinaria. $4^{a}$ ed. Ciudad de México, El Manual Moderno, 2012.

Fargen, K. M. \& Hoh, B. L. The debate over eponyms. Clin. Anat., 27(8):1137-40, 2014.

Federative Committe on Anatomical Terminology (FCAT). Terminologia Anatomica. International Anatomical Terminology. Stuttgart, Thieme, 1998.

Hesíodo. Teogonía. Barcelona, Gredos, 2010

International Committee on Veterinary Gross Anatomical Nomenclature (ICVGAN). Nomina Anatomica Veterinaria. $6^{\text {th }}$ ed. Knoxville, Editorial Committee, 2017. 
Kayalvizhi, I.; Bansal, S.; Dhidharia, K.; Narayan, R. K. \& Kumar, P. Morphometric study of the articular facets of atlas vertebra in north Indian population. Int. J. Anat. Res., 5(2.2):3829-32, 2017.

Liebich, H. G. \& König, H. Esqueleto axial. En: König, H. \& Liebich, H. G. Anatomía de los Animales Domésticos. Tomo I. Aparato locomotor. $2^{\mathrm{a}}$ ed. Madrid, Médica Panamericana, 2005.

Manfreda, E.; Mitteroecker, P.; Bookstein, F. L. \& Schaefer, K. Functional morphology of the first cervical vertebra in humans and nonhuman primates. Anat. Rec. B New Anat., 289(5):184-94, 2006.

Pollucis, I. Onomasticon. Lipsiae, Libraria Kuenhiana. 1824.

Riascos Catañeda, R. \& Castro Navarro, J. C. Etimología de los hueso del cuerpo humano. Repert. Med. Cir., 13 (4):184-6. 2004.

Roa, I. Agenesia parcial del arco posterior del atlas. Reporte de caso. Rev. Argent. Anat. Onlin), 6(3):113-6, 2015.

Sánchez-García, E. \& Caro, L. Variantes Anatómicas y Anomalías en la Columna Cervical y sus Implicaciones Clínicas. En: Alonso, S.; Hervella, M.; Izaguirre, N.; Peña, J. A.; Rebato, E. \& De la Rúa, C. La Investigación en Antropología Física. Una Mirada al Futuro. Bilbao, Servicio Editorial de la Universidad del País Vasco, 2014. pp.199-216.

van Dijk, C. N.; van Sterkenburg, M. N.; Wiegerinck, J. I.; Karlsson, J. \& Maffulli, N. Terminology for Achilles tendon related disorders. Knee Surg. Sports Traumatol. Arthrosc., 19(5):835-41, 2011.

Vargas Sanabria, M. Anatomía y exploración física de la columna cervical y torácica. Med. Leg. Costa Rica, 29(2):77-92, 2012.

Vesalius, A. De Humani corporis Fabrica. Basileae, Libri feptem, 1555.

Yamahata, H.; Hirano, H.; Yamaguchi, S.; Mori, M.; Niiro, T.; Tokimura, H. \& Arita, K. What Is the Most Representative Parameter for Describing the Size of the Atlas? CT Morphometric Analysis of the Atlas with Special Reference to Atlas Hypoplasia. Neurol. Med. Chir. (Tokyo), 57(9):461-6, 2017.

\author{
Dirección para correspondencia: \\ Jorge Eduardo Duque Parra \\ Departamento de Ciencias Básicas \\ Programa de Medicina \\ Universidad de Caldas \\ Sede principal: Calle 65 No. 26-10. \\ Manizales \\ COLOMBIA
}

Email: jduqueparra@yahoo.com.mx.

Recibido : 05-09-2018

Aceptado : 27-12-2018 